\title{
Energy Efficient Trade-off between Communication and Sensing in Wireless Gas Sensor Node
}

\author{
Andrey Somov ${ }^{1}$, Mikhail Ivanov ${ }^{2}$, Alexander Baranov², Alexey Savkin², \\ and Sithamparanathan Kandeepan ${ }^{1}$ \\ ${ }^{1}$ CREATE-NET, \\ Networks and Services area, \\ via alla Cascata 56/D, 38123 Trento (TN), Italy \\ [andrey.somov; kandee] dereate-net.org \\ ${ }^{2}$ Moscow State Aviation Technological University, \\ Department of High Tech Electronics, \\ Orshanskaya 3, 121552 Moscow, Russia \\ radio@mati.ru
}

\begin{abstract}
A wireless sensor network is a distributed collection of resource constrained tiny nodes capable of operating with minimal user attendance. Communication circuit is normally the most power consuming part of sensor node. Depending on the application, the node may have on board various extra hardware components with even higher power consumption. These components can impose restrictions in terms of providing the same communication range without increasing power consumption. In this paper we investigate the energy efficient balance between the communication and sensing modes in a gas sensor node. First, we analyze how to secure the energy efficient sensing of environment using the developed framework. Then we establish the efficient transmission power rate for each sensing power level. Finally, the effective distances for the appropriate transmission rates are defined.
\end{abstract}

Keywords: energy efficient communication, wireless sensor node, power management, gas sensor.

\section{Introduction}

A Wireless Sensor Network (WSN) consists of wireless nodes which measure physical conditions using sensors (i.e., temperature, humidity, vibration, pressure), digitize it and keep or distribute the measured data over the network. Due to their flexibility and low cost, WSNs have recently become widely applied in traffic regulation, fire alarm in buildings, wild fire monitoring, agriculture, health monitoring, entertainment, building energy management, and ecological monitoring [1], [2].

WSNs provide the opportunity to build autonomous systems without the need to wire up an entire network, thus progressively replacing wired embedded systems. 
According to the application requirements, the wireless sensor nodes can be deployed over a large area or can be amassed in a small section for the specific data measurements.

Due to the fact that the wireless sensor node is energy constrained device, it must operate using power efficient hardware and software technologies to increase its lifetime. Energy scavenging technology [3] can improve the sensor node lifetime and decrease the cost of the network maintenance: the nodes are usually powered by AA cells, but the batteries must be replaced when exhausted. According to OnWorld Research the battery replacement cost will be $\$ 1$ Billion in 2013 [4]. Energy scavenging techniques [4], [5], [6], [7], [8], [9] are aimed at decreasing or even canceling the network maintenance cost.

Radio chip is normally the most power-hungry component of sensor node [10]. In last years, several strategies [11] were developed to decrease the power consumption of communication circuit e.g., optimal synchronization [12], adaptive duty cycling [13], data aggregation technique [14], sleep scheduling [15]. However, if a wireless node has an on-board powerful sensor (see Table 1) a power management sensing technique is also required [16], [17], [18].

In this paper we investigate the energy efficient balance between the sensing and communication modes in the gas sensor node with a powerful catalytic gas sensor. This platform was proposed in our previous work [19]. Balancing is based on the identification of energy efficient sensing mode with the subsequent computation of energy efficient communication. The resultant trade-off enables us to define the effective distance between the transmitter and the receiver for each of the corresponding power transmission rates.

The paper is organized as follows. Section 2 will introduce the reader to the aspects of energy efficient sensing and communication: the overview of the developed framework for the efficient sensing evaluation and a concept of the wireless gas sensor platform are presented in subsection 2.1, and the transmitter-receiver separation distance is analyzed in subsection 2.2. The results obtained for the energy efficient balancing are presented in Section 3. Finally, we discuss prospects of our work and conclude.

\section{Background}

A WSN contains a number of sensor nodes connected by a radio channel. A typical sensor node block diagram is presented in Figure 1.

Typical sensor node has four main blocks on board: a Central Processing Unit (CPU), memory, Input-Output (I/O) ports and radio. Depending on the application, the sensor node may have various extra hardware components on board. Table 1 and Table 2 present popular transceivers and sensors respectively.

Table 1 shows the sensors with typical sensing functions available in the market. Table 2 summarizes some popular transceivers which used by sensor nodes. The communication part of a sensor node is usually the main energy sink [10]. In fact, if transmission takes less time than acquisition, some sensors may consume more energy than the transceiver. 


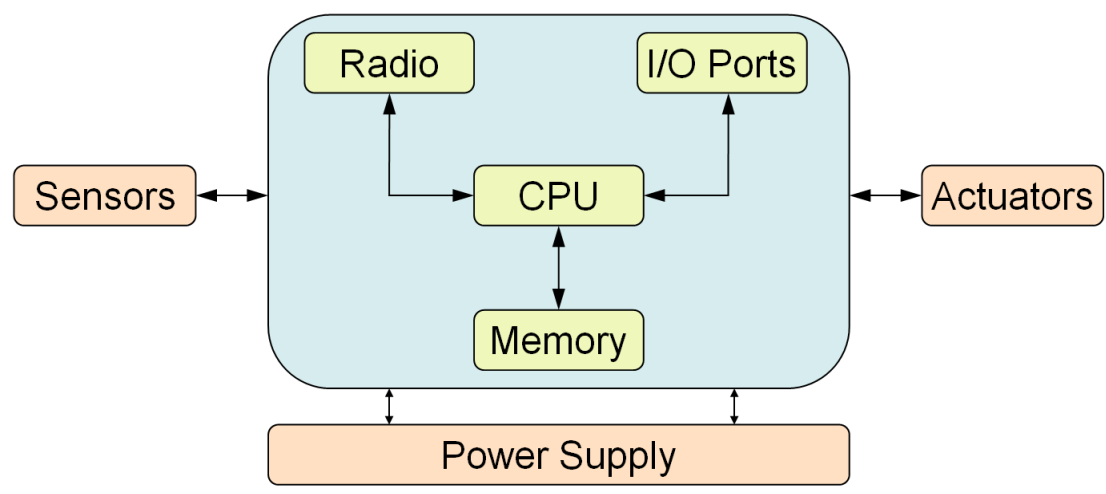

Fig. 1. Sensor node anatomy.

Table 1. Power consumption for some off-the-shelf sensors with typical functions.

\begin{tabular}{llll}
\hline Sensor & Manufacturer & Purpose & $\begin{array}{l}\text { Power } \\
\text { consumption, mW }\end{array}$ \\
\hline STDS75 & STM & Temperature & 0.5 \\
MMA7330L & Freescale & Accelerometer (x, y, z) & 1.4 \\
HIH-4000-001 & Honeywell & Humidity & 10 \\
B6TS series & Omron & Touch & 16 \\
HAF series & Honeywell & Air flow & 23 \\
2200/2600 series & Gems & Pressure & 50 \\
TGS2610 & FIGARO & LP gas & 280 \\
CP18, VL18, & Visolux & Proximity & 350 \\
GM60, GLV30 & & & \\
NAP-66A & Nemoto & Flammable gases & 360 \\
MC series & Hanwei Electronics & Combustible gases & $420,450,600$ \\
AD81 & GE & Gasoline \& diesel & 620 \\
& & exhaust gas & \\
MQ-4 & Hanwei Electronics & Methane, natural gas, smoke & 750 \\
\hline
\end{tabular}

Table 2. Power consumption of popular transceivers for wireless sensor nodes.

\begin{tabular}{|c|c|c|c|}
\hline Transceiver & Manufacturer & $\mathbf{T x}, \mathbf{m W}$ & $\mathbf{R x}, \mathbf{m W}$ \\
\hline TR1000 & RF Monolithics & $36 \mathrm{~mW}($ at $0 \mathrm{dBm})$ [16] & $9 \mathrm{~mW} \quad[16]$ \\
\hline CC1000 & Texas Instruments & $42($ at $0 \mathrm{dBm})$ [16] & $29[16]$ \\
\hline JN5148-001-M00/03 & Jennic & 45 (at $+2.5 \mathrm{dBm})$ & $52.5^{\circ}$ \\
\hline $\mathrm{CC} 2420$ & Texas Instruments & 52.2 (at $0 \mathrm{dBm})$ & 56.4 \\
\hline ETRX3 & Telegesis & $93($ at $+3 \mathrm{dBm})$ & $\begin{array}{l}75 \text { (at } 12 \mathrm{MHz} \\
\text { clock speed) }\end{array}$ \\
\hline ETRX2 & Telegesis & 106.5 (at $+3 \mathrm{dBm})$ & 106.5 \\
\hline JN5139-xxx-M00/01.03 & Jennic & $111(\mathrm{at}+2.5 \mathrm{dBm})$ & 111 \\
\hline
\end{tabular}

In this paper we consider a wireless gas sensor node with a gas sensor on board. The power consumption parameters of this node are shown in Table 3. 
Table 3. Power consumption of the wireless gas sensor node.

\begin{tabular}{cccc}
\hline \multirow{2}{*}{ Mode } & \multicolumn{3}{c}{ Power consumption, $\mathrm{mW}$} \\
\cline { 2 - 4 } & Processing & Sensing circuit & Communication \\
\hline Gas measurement & 7.59 & 140 & 0.03 \\
Data transmission & 7.59 & 0 & $75(\mathrm{Rx})$ \\
Energy saving & 0.06 & 0 & 0.03 \\
\hline
\end{tabular}

\subsection{Energy Efficient Sensing: Framework}

The framework developed for evaluating power consumption of the gas sensor node during sensing is shown in Figure 2.

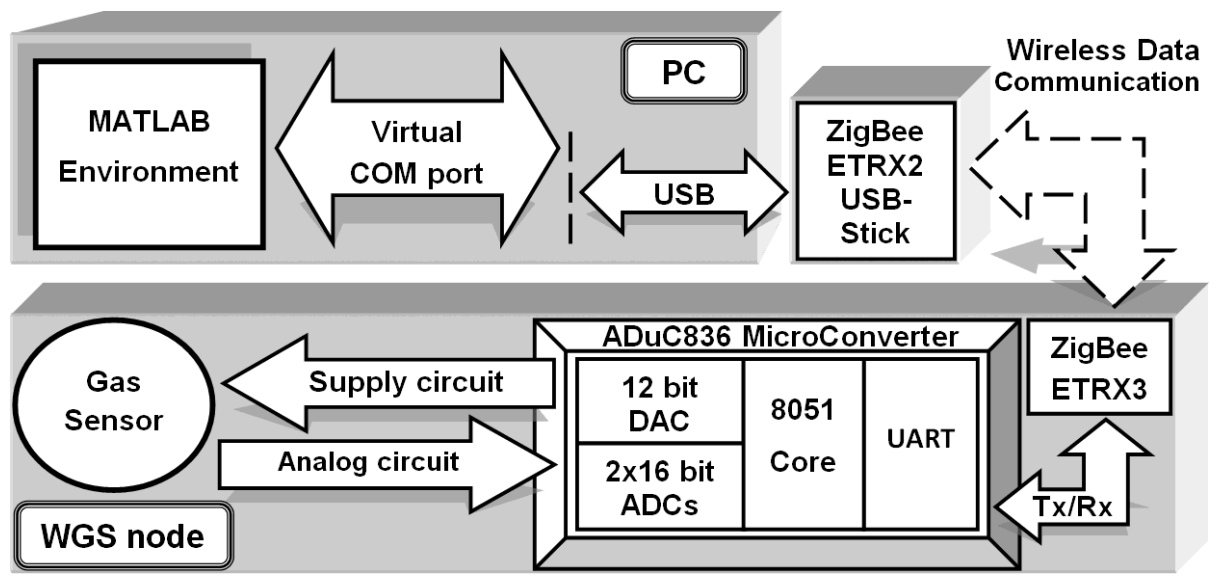

Fig. 2. Framework block diagram.

The framework consists of two parts: Wireless Gas Sensor (WGS) node shown in Figure 3 and a Matlab Application (MA). WGS node and MA communicate via wireless channel implemented by ETRX3 module and USB stick [20] with ZigBee standard [21]. Wireless channel is organized between the USB stick with transceiver plugged in PC and transceiver installed on WGS node.

WGS node includes the 8-bit Microcontroller Unit (MCU) ADuC836, the wireless modem ETRX3 and two gas sensors as the essential units. MCU has the 8051 core, two 16-bit $\sum-\Delta$ analog-to-digital converters, the 12-bit digital-to-analog converter and several power saving modes. The ETRX3 low power $2.4 \mathrm{GHz}$ modem supports ZigBee/802.15.4 standard with $250 \mathrm{~kb} / \mathrm{s}$ data rate. The ADP3335 regulator stabilizes power supply of the WGS node up to $3.3 \mathrm{~V}$. To accomplish wireless connection, MCU sends initialization commands to ETRX3 via UART interface with $115200 \mathrm{~b} / \mathrm{s}$ speed. After successful initialization WGS node connects to PC via wireless channel in Data mode with $250 \mathrm{~kb} / \mathrm{s}$ data rate. In order to interface a USB port and Matlab environment on PC, the virtual COM port is realized. 


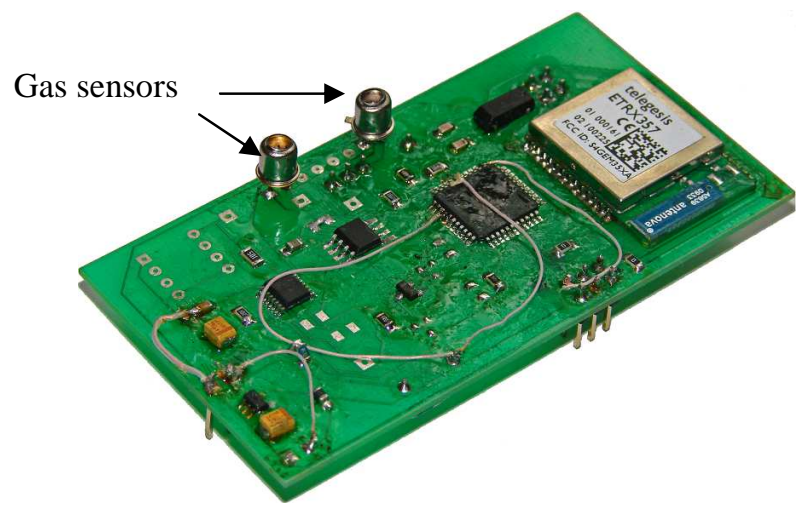

Fig. 3. The wireless gas sensor node $(80 \times 45 \mathrm{~mm})$.

The framework operates in the following way. The application in Matlab environment initiates the measurement procedure by sending directives to WGS node. ETRX3 transmits these commands to MCU which determines the appropriate heating profile for the sensing circuit. Finally, WGS node sends the sensing results back to the Matlab application for a thorough analysis and real-time representation in the form of graphs.

\subsubsection{Test case}

As a test case, we have investigated the sensing capabilities of the catalytic gas sensor DTK-2 [22] (see Figure 3). Its average power consumption in Sensing mode is 60-70 $\mathrm{mW}$.

In order to operate in the energy efficient mode during scanning of environment, the sensors apply constant current signals to warm up the sensing layer. The main purpose of this experiment is therefore gaining the sensor's response in the period from power-up to operating mode activation.

For the experiment, we have placed the WGS node with DTK-2 sensors into the gas chamber made of radio transparent covering. The PC with Matlab application has enabled us to remotely manage the WGS node. Various heating profiles have been tested in the air and in the presence of $0.25 \%$ and $2 \%$ methane $\left(\mathrm{CH}_{4}\right)$.

Figure 4 shows the DTK-2 sensor's response in the absence and presence of $\mathrm{CH}_{4}$ of different density and at the 2.8 voltage of heating pulse $\left(U_{p}\right)$. To ensure the higher resolution of the sensor, a longer period of heating the sensor is required. Thus, for example, the threshold of $2 \% \mathrm{CH}_{4}$ can be detected with 0.35 seconds of heating, but for the reliable $0.25 \% \mathrm{CH}_{4}$ threshold detection the module needs a period by a factor of 1.5 longer. The solution of this dilemma is a trade-off between resolution and energy efficiency.

DTK-2 sensor may detect $0.25 \% \mathrm{CH}_{4}$ threshold at $2.4 \mathrm{~V}$ of $U_{p}$ (see Figure 5) only when the heating circuit will spend $2.5 \mathrm{~mJ}$ for $0.8 \mathrm{~s}$. However, this value is $2.0 \mathrm{~mJ}$ for $0.5 s$ when $U_{p}$ is $2.8 \mathrm{~V}$. 


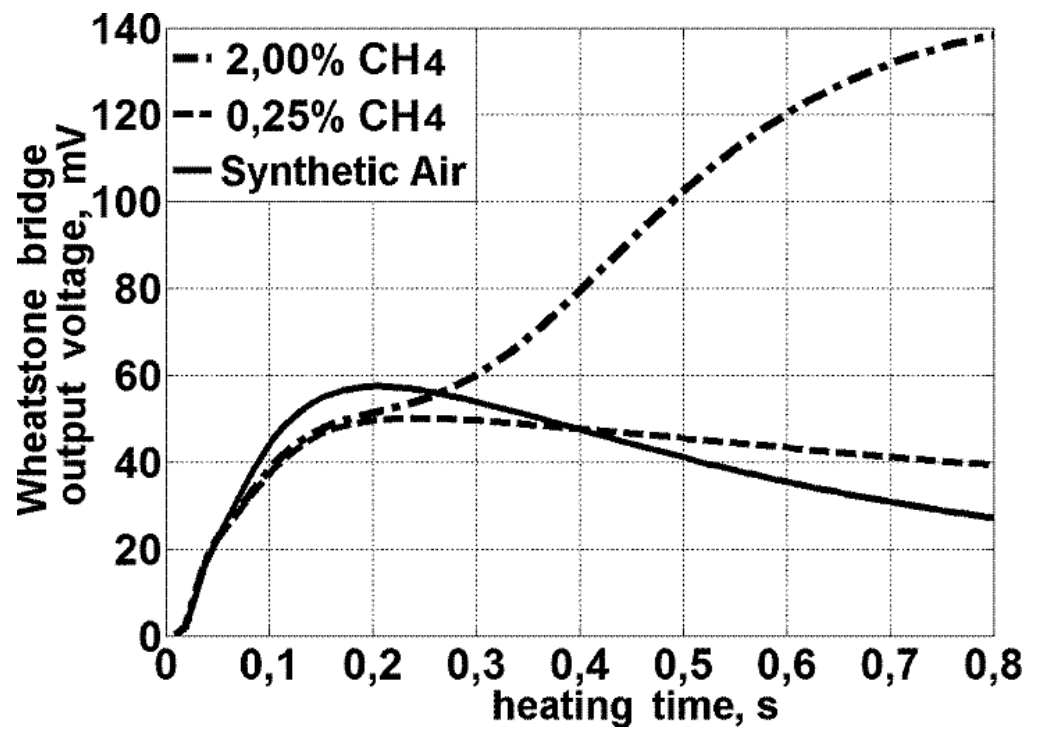

Fig. 4. DTK-2 sensor characteristics at $U_{p}=2.8 \mathrm{~V}$.

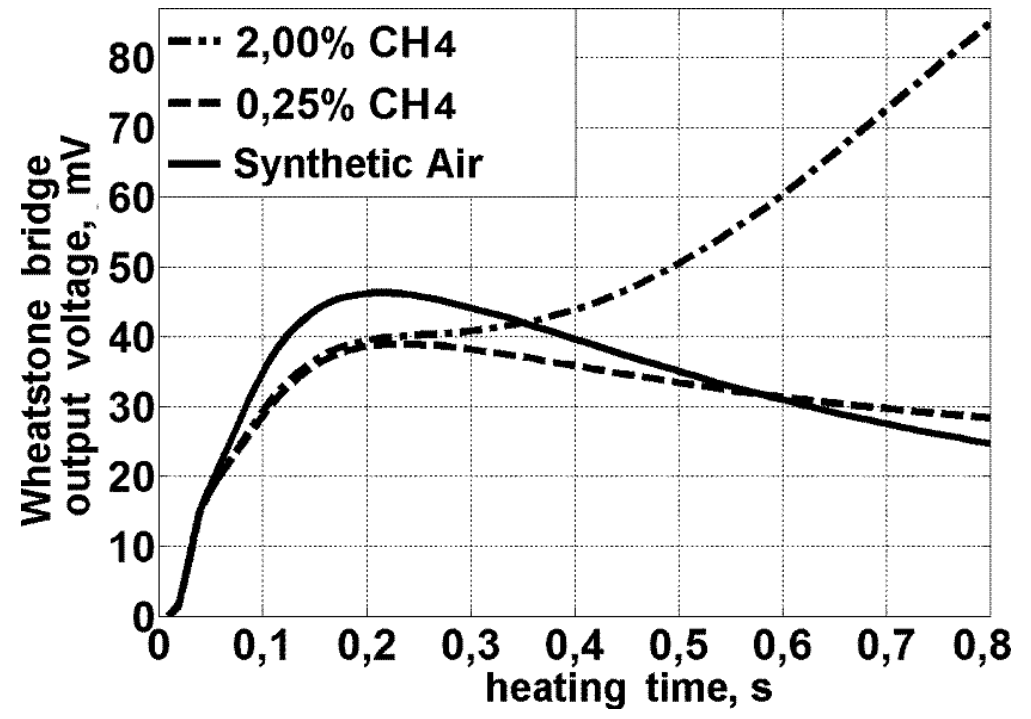

Fig. 5. DTK-2 sensor characteristics at $U_{p}=2.4 \mathrm{~V}$. 


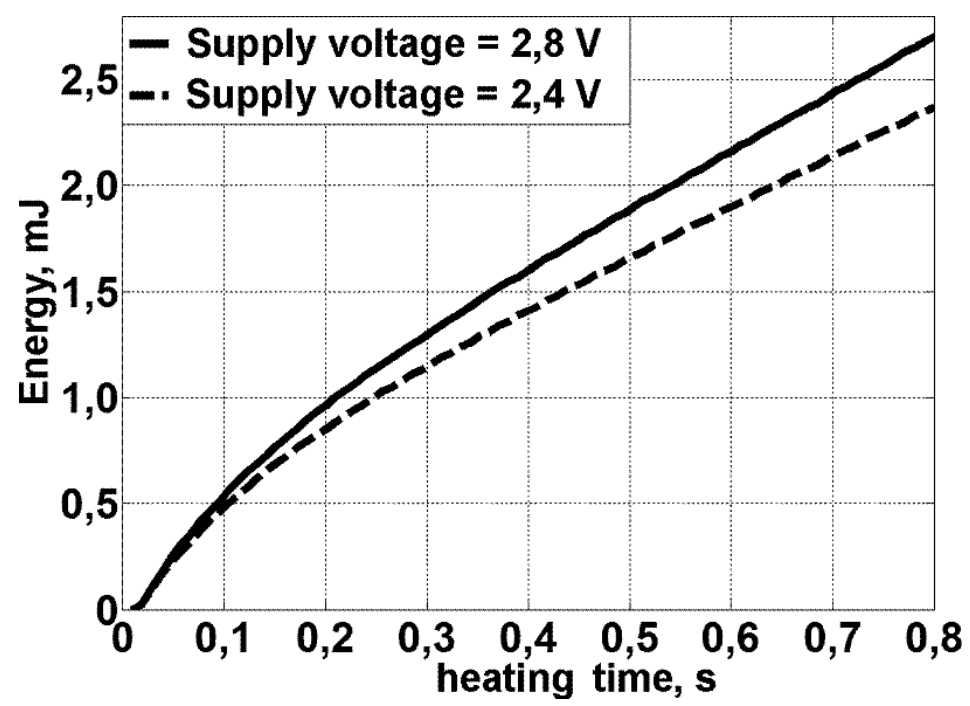

Fig. 6. Energy consumption of the sensing circuit at different supply voltage $\left(U_{p}\right)$.

One may conclude from the above that the decrease of supply voltage $\left(U_{p}\right)$ does not result in the increase of energy efficiency of sensing circuit (see Figure 6), but increases its power-up time and a rise in energy dissipation.

\subsection{Communication}

In this section we investigate how the Transmitter-Receiver (T-R) separation distance (d) depends on transmission power $\left(P_{T}\right)$ which in our case depends on the power consumed by the sensor $\left(P_{S}\right), P_{T}$ and $P_{S}$ are connected by (4) through the total energy $(E)$ stored in an energy buffer.

We assume that a receiver gets the power sent by transceiver minus loss power:

$$
P_{R}(d)[d B W]=P_{T}[d B W]-P L(d)[d B]
$$

where $P_{R}$ is a power collected by receiver, $P_{T}$ is a transmission power and $P L$ is the path loss for an arbitrary T-R separation which is expressed as follows:

$$
\overline{P L}(d B W)=\overline{P L}\left(d_{0}\right)+10 \alpha \log \left(\frac{d}{d_{0}}\right)
$$

where $d_{0}$ is the reference distance, $\alpha$ is the path loss exponent (we use $\alpha=4.5$ for outdoor applications, see Table 3.2 in [23]) and $d$ is the T-R separation distance. 


$$
\overline{P L}\left(d_{0}\right)=\left(\frac{4 \pi d_{0} f_{c}}{c}\right)^{2}
$$

where $f_{c}$ is the transmit frequency $(2.4 \mathrm{GHz})$ and $c$ is the speed of light.

\section{Energy Balancing between Communication and Sensing}

To find an energy-efficient trade off between communication and sensing we examine a periodic application where a gas sensor node scan the environment and sends the data to the gate each 20 minutes.

The gas sensor node`s overall energy consumption is determined by energy used during sensing, $E_{S}$, transmitting, $E_{T}$, and processing the data, $E_{P}$.

$$
E=E_{S}+E_{T}+E_{P}
$$

where

$$
E_{T}=I_{T} \cdot V \cdot t_{\text {packet }}
$$

and

$$
E_{P}=I_{P} \cdot V \cdot t_{\text {proc }}
$$

Table 4 lists the parameters for a monitoring application. Parameters $E_{s}, t_{\text {packet }}, t_{\text {proc }}$, are derived from the experiments. Under packet processing time we mean the time required for $\mathrm{MCU}$ to process the data and send them to the transmitter.

Table 4. Parameters for a monitoring application.

\begin{tabular}{lll}
\hline Notation & Parameter & Value \\
\hline $\mathrm{E}_{\mathrm{s}}$ & Energy consumption during sensing $(\mathrm{mJ})$ & $2.0(2.8 \mathrm{~V}), 2.5(2.4 \mathrm{~V})$ \\
$\mathrm{C}_{\text {batt }}$ & Capacity of battery (mAh) & 2200 \\
$\mathrm{~V}$ & Node operating voltage $(\mathrm{V})$ & 3 \\
$\mathrm{R}$ & Data rate $(\mathrm{kB} / \mathrm{s})$ & 250 \\
$\mathrm{~L}_{\text {packet }}$ & Packet (data \& overhead) length (byte) & 47 \\
$\mathrm{t}_{\text {packet }}$ & Packet transmission time (us) & 1504 \\
$\mathrm{t}_{\text {proc }}$ & Packet processing time (ms) & 5 \\
$\mathrm{I}_{\mathrm{T}}$ & Transmission current $(\mathrm{mA})$ & $28.5(0 \mathrm{dBm}), 31(3 \mathrm{dBm}), 42(8 \mathrm{dBm})$ \\
$\mathrm{I}_{\mathrm{P}}$ & Processing current $(\mathrm{mA})$ & 2.3 \\
\hline
\end{tabular}


The lifetime of the gas sensor node, $t_{l}$, depends on the battery capacity, $C_{\text {batt }}$, and the overall energy consumed. With (7) we bound the node lifetime by the available battery capacity, $E_{s}=2.5 \mathrm{~mJ}$ and data transmission rate with $0 \mathrm{dBm}$.

$$
t_{l}=\frac{C_{\text {batt }} \cdot V}{E} \cdot 3600
$$

Figure 7 shows the relationship between the transmission power and the sensing power. The power inefficient sensing mode at the $3.125 \mathrm{~mW}$ level (in terms of energy) may ensure the packets transmission at $85.5 \mathrm{~mW}$. However, in the case of switching the MCU over to power efficient sensing mode (in terms of energy), it allows the packets transmission at $103 \mathrm{~mW}$.

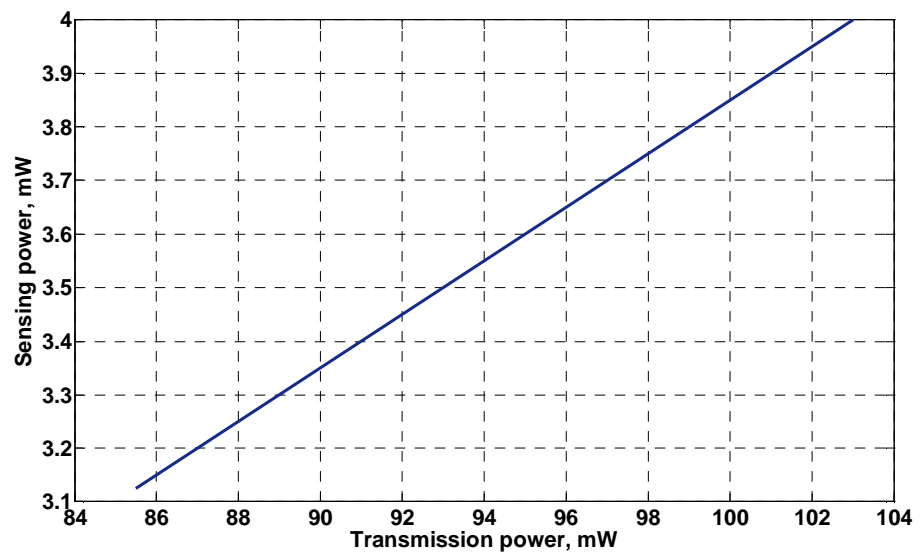

Fig. 7. Power transmission and power sensing dependence.

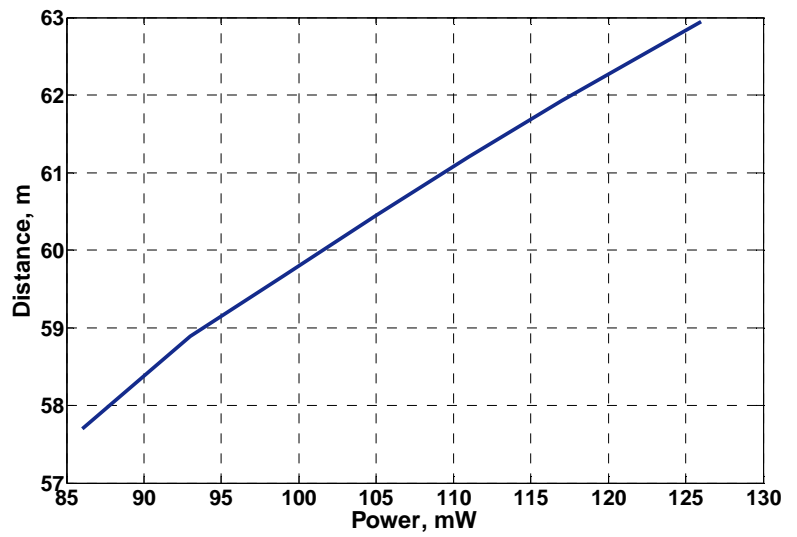

Fig. 8. Effective distance for the transmitting power. 
Using (1)-(3) and the data from ETRX3 Zigbee module specification [20] we may define how the T-R separation distance depends on the transmission power (see Figure 8). If the gas sensor node operates in the energy inefficient sensing mode, the radio circuit transmits the packet over approximately $57 \mathrm{~m}$. In the energy efficient sensing mode this distance increases up to $60 \mathrm{~m}$.

\section{Conclusion and Future Work}

The present work has been aimed at investigating the energy efficient trade-off between the sensing and communication modes through applying WSN paradigm. We have built a wireless gas sensor node used as an experimental test-bed, and have created a Matlab application. With this framework we have managed to define the energy-efficient mode for environment sensing with the powerful DTK-2 gas sensor. Based on the lifetime model for the sensor node, we have established the balanced power consumption required for the communication circuit.

Besides, using a path loss model, we have found the maximum effective distance between the transmitter and the receiver which corresponds to the transmission power rate.

Our prospective work aims at conducting real experiments for the effective communication range evaluation and the system deployment. To begin with, we intend to deploy the system using facilities of the Department of High Tech Electronics at Moscow Aviation Technological University. Since the platform supports the energy scavenging technology (see our previous work [19]), we are also planning to implement the system deployment in real conditions.

Acknowledgments: The authors would like to acknowledge the Russian Federal Program "Development of Electronic Components and Radio Electronics" Grant No. 01.426.11.0022, and the C2POWER project under the EU-FP7 framework program (ICT project ICT-248577) sponsored by the European Commission.

\section{References}

1. Chong, C.-Y., Kumar, S. P.: Sensor Networks: Evolution, Opportunities, and Challenges. In Proceedings of the IEEE, 91 (8), pp. 1247--1256 (2003)

2. Somov, A., Sachidananda, V., and Passerone, R.: A Self-Powered Module with Localization and Tracking System for Paintball. In: Hummel, K.A. and Sterbenz, J.P.G. (eds.) IWSOS 2008. LNCS, vol. 5343, pp. 182--193. Springer, Heidelberg (2008)

3. Roundy, S., Wright, P. K., and Rabaey, J. M.: Energy Scavenging for Wireless Sensor Networks. Kluwer Academic Publishers Group (2003)

4. Cymbet white paper: Zero Power Wireless Sensors, www.cymbet.com (2009)

5. Dutta, P., Hui, J., Jeong, J., Kim, S., Sharp, C., Taneja, J., Tolle, G., Whitehouse, K., and Culler, D.: Trio: Enabling Sustainable and Scalable Outdoor Wireless Sensor Network Deployments. In Fifth International Conference on Information Processing in Sensor Network, pp. 407--415 (2006) 
6. Torah, R., Glynne-Jones, P., Tudor, M., O`Donnell, T., Roy, S., and Beeby, S.: Self-Powered Autonomous Wireless Sensor Node Using Vibration Energy Harvesting. J. Measurement Science and Technology. 19 (12), pp. 1--8 (2008)

7. Chee, Y. H., Koplow, M., Mark, M., Pletcher, N., Seeman, M., Burghardt, F., Steingart, D., Rabaey, J., Wright, P., and Sanders. S.: Picocube: A $1 \mathrm{~cm}^{3}$ Sensor Node Powered by Harvested Energy. In Design Automation Conference, pp. 114--119 (2008)

8. Park, C., and Chou, P. H.: Ambimax: Autonomous Energy Harvesting Platform for Multisupply Wireless Sensor Nodes. In the Third Annual IEEE Communications Society on Sensor and Ad Hoc Communications and Networks, pp. 168--177 (2006)

9. Phipps, A., Liu, F., Cattafesta, L., Sheplak, M., and Nishida, T.: Demonstration of a Wireless, Self-Powered, Electroacoustic Liner System. J. Acoustic Society of America. 125 (2), pp. 873--881 (2009)

10. Crossbow wireless modules: MICAz, MICA2, TelosB, www.xbow.com (2010)

11. Anastasi, G., Conti, M., Di Franceso, M., and Passarella, A.: Energy Conservation in Wireless Sensor Networks: a Survey. J. Ad Hoc Networks 44 (4), pp. $537--568$ (2009)

12. Macii, D., Ageev, A., Somov, A.: Power Consumption Reduction in Wireless Sensor Networks through Optimal Synchronization. In the International Instrumentation and Measurement Technology Conference, pp. 1346--1351 (2009)

13. Hsu, J., Zahedi, S., Kansal, A., Srivastava, M., and Raghunathan, V.: Adaptive duty cycling for energy harvesting systems. In Proceedings of the International Symposium on Low Power Electronics and Design, pp. 180--185 (2006)

14. Croce, S., Marcelloni, F., and Vecchio, M.: Reducing Power Consumption in Wireless Sensor Networks Using a Novel Approach to Data Aggregation. The Computer Journal (51), pp. 227--239 (2007)

15. Subramanian, R. and Fekri, F.: Sleep Scheduling and Lifetime Maximization in Sensor Networks: Fundamental Limits and Optimal Solutions. In Proceedings of the $5^{\text {th }}$ International Conf on Information Processing in Sensor Networks, pp. 218--225 (2006)

16. Alippi, C., Anastasi, G., Di Francesco, M., and Roveri, M.: Energy Management in Wireless Sensor Networks with Energy-Hungry Sensors. IEEE Instrumentation and Measurement Magazine. 12 (2), 16--23 (2009)

17. Kim, N., Choi, S., and Cha, H.: Automated Sensor-Specific Power Management for Wireless Sensor Networks. In Proceedings of IEEE Conference on Mobile Ad Hoc and Sensor Systems, pp. 305--314 (2008)

18. Willet, R., Martin, A., and Nowak, R.: Backcasting: Adaptive Sampling for Sensor Networks. In Proc of Information Processing in Sensor Networks, pp. 124--133 (2004)

19. Somov, A., Spirjakin, D., Ivanov, M., Khromushin, I., Passerone, R., Baranov, A., and Savkin, A.: Combustible Gases and Early Fire Detection: an Autonomous System for Wireless Sensor Networks. In the Proceedings of the First International Conference on Energy-Efficient Computing and Networking, pp. 85--93 (2010)

20. ETRX3 ZigBee module by Telegesis: http://telegesis.com (2010)

21. IEEE Computer Society: 802.15.4 Wireless Medium Access Control and Physical Layer Specifications for Low-Rate Wireless Personal Area Networks, www.ieee.org (2003)

22. Vasiliev, A., Pavelko, R., Gogish-Klushin, S., Kharitonov, D., Gogish-Klushina, O., Pisliakov, A., Sokolov, A., Samotaev, N., Guarnieri, V., Zen., M., and Lorenzelli, L.: Sensors Based on Technology "Nano-on-Micro" for Wireless Instruments Preventing Ecological and Industrial Catastrophes. Sensors for Environment, Health and Security, pp. 205--227. Springer, Netherlands (2008)

23. Rappaport, T.S.: Wireless Communications: Principles \& and Practice. Prentice Hall PTR, New Jersey (1996) 